\title{
Cell Culture Systems of HCV Using JFH-1 and Other Strains
}

\author{
Takaji Wakita \\ National Institute of Infectious Diseases, Tokyo 162-8640, Japan \\ Correspondence: wakita@nih.go.jp
}

Hepatitis C virus (HCV) infection is seen worldwide and is a significant cause of severe chronic liver diseases. Recently, a large number of direct-acting antivirals (DAAs) have been developed against HCV infection, resulting in significant improvements in treatment efficacy. Rapid progress in HCV research has been largely dependent on the development of HCV culture systems and small animal infection models. In the development of HCV cell culture systems, the discovery of the JFH-1 clone, an HCV strain isolated from a fulminant hepatitis $\mathrm{C}$ patient, was a key finding. The JFH-1 strain was the first infectious HCV strain belonging to genotype 2a. JFH-1 replicated efficiently in cultured cell lines without acquiring adaptive mutations, providing the secretion of infectious viral particles into the culture medium. Recently, other HCV strains also were reported to be infectious in cultured cells with adaptive viral mutations, but genotype- $1 \mathrm{~b}$ infectious HCV clones and virus culture systems for clinical isolates are still missing. These infectious HCV systems have provided powerful tools to study the viral life cycle, to construct antiviral strategies, and to develop effective vaccines.

$\mathrm{H}^{2}$ epatitis C virus (HCV) was first identified in 1989 as a principal agent in posttransfusion and sporadic acute hepatitis (Choo et al. 1989; Kuo et al. 1989). Sequence analysis revealed that $\mathrm{HCV}$ is a plus-stranded RNA virus that belongs to the family Flaviviridae and genus Hepacivirus. The HCV RNA genome is $\sim 9.6 \mathrm{~kb}$ long and contains a single long open reading frame of $\sim 3000$ codons (Fig. 1). Most of the classic Flaviviruses cause mosquito-borne infections. However, HCV causes blood-borne infection and chronic liver disease and thus is classified as a hepatitis virus. It has not been clear how the classic vector-borne flaviviruses and hepaciviruses evolved. Recently, several animal hepaciviruses were identified from horses and other animals (Hartlage et al. 2016). The analysis of animal hepaciviruses is expected to provide further evolutionary information about HCV.

Following the discovery of HCV, the frequency of new posttransfusion HCV infections was significantly reduced in many countries by implementation of HCV screening of blood products. Most HCV infections cause persistent viral infection resulting in chronic hepatitis, liver cirrhosis, and hepatocellular carcinoma; however, highly effective anti-HCV treatment has been developed by using direct-acting antivirals (DAAs). DAA treatment has achieved a high success rate of sustained virological response

Editors: Arash Grakoui, Jean-Michel Pawlotsky, and Glenn Randall

Additional Perspectives on Hepatitis C Viruses: The Story of a Scientific and Therapeutic Revolution available at www.perspectivesinmedicine.org

Copyright (C 2019 Cold Spring Harbor Laboratory Press; all rights reserved; doi: 10.1101/cshperspect.a036806

Cite this article as Cold Spring Harb Perspect Med 2019;9:a036806 
T. Wakita



Figure 1. Hepatitis C virus (HCV) genome structure. HCV RNA genome is $~ 9600$ nt long. The virus RNA contains a single long open reading frame (ORF) of $\sim 3000$ codons long that is flanked by $5^{\prime}$ and $3^{\prime}$ untranslated regions (UTRs) with secondary RNA structures. At least 10 viral proteins are processed from the large polypeptides encoded by the ORF. Each viral protein has a single or multiple functions. The NS3, NS5A, and NS5B proteins are the targets of currently available direct-acting antivirals (DAAs).

among treated patients, thereby reducing the risk of advanced liver disease.

Detailed analysis of the viral life cycle of HCV using virus culture has been critical (Fig. 2A). Several HCV clones have been reported to be infectious in chimpanzees; however, the propagation of HCV in cultured cells remained a challenge for several years (Yanagi et al. 1997, 1999; Bartenschlager and Lohmann 2001; Lanford et al. 2001; Bukh et al. 2002). This limitation may have been the result of a number of factors, including a low replication capacity of the virus to establish persistent infection and tropism of the virus for highly differentiated hepatocytes. Inoculation of patient sera or plasma into cultured cells results in only a limited level of HCV replication, as determined by nested reverse transcription-polymerase chain reaction (RTPCR). However, Lohmann et al. (1999) were the first to report efficient replication of an HCV subgenomic replicon by replacement of an HCV structural region (with or without the NS2 gene) with a neomycin-resistance-encoding gene (Fig. 3A). Transfection of replicon RNA into Huh7 hepatocellular carcinoma cells, followed by G418 selection culture for several weeks, permitted the establishment of replicons; robust replication of viral RNA was observed in these cells (Fig. 3B). Analysis of the established replicon cells revealed the presence of adaptive mutations in most of the replicon genomes. These mutations enhanced viral replication to different degrees, and some combinations of these lesions were observed to strongly enhance replication (Blight et al. 2000; Krieger et al. 2001; Lohmann et al. 2001). This replicon system proved to be a great advantage for the detailed analysis of viral RNA translation and replication mechanisms, and permitted the identification of host factors required for replication in cell culture (Fig. 2A). However, the most important contribution of the replicon system was the development of DAAs with activity against $\mathrm{HCV}$. The activities of DAAs targeting the NS3/4A protease, the NS5A protein, and the NS5B RNA polymerase were validated using the replicon system before testing in clinical trials.

Notably, the construction of the subgenomic replicon genome required the deletion of $\mathrm{HCV}$ structural genes, meaning that viral particles were not produced from the replicon cells. To establish an HCV infection system, genomic replicons containing a structural region with adaptive mutations in a nonstructural region also were generated; these constructs showed efficient replication in transfected Huh7 cells (Ikeda et al. 2002; Pietschmann et al. 2002; Blight et al. 2003). Unfortunately, these genomic replicons still did not produce viral particles. Furthermore, a full-length viral RNA genome (harboring adaptive mutations) that had been synthesized in vitro still was not infectious in chimpanzee, in contrast to the wild-type genome (Bukh et al. 2002). 
A

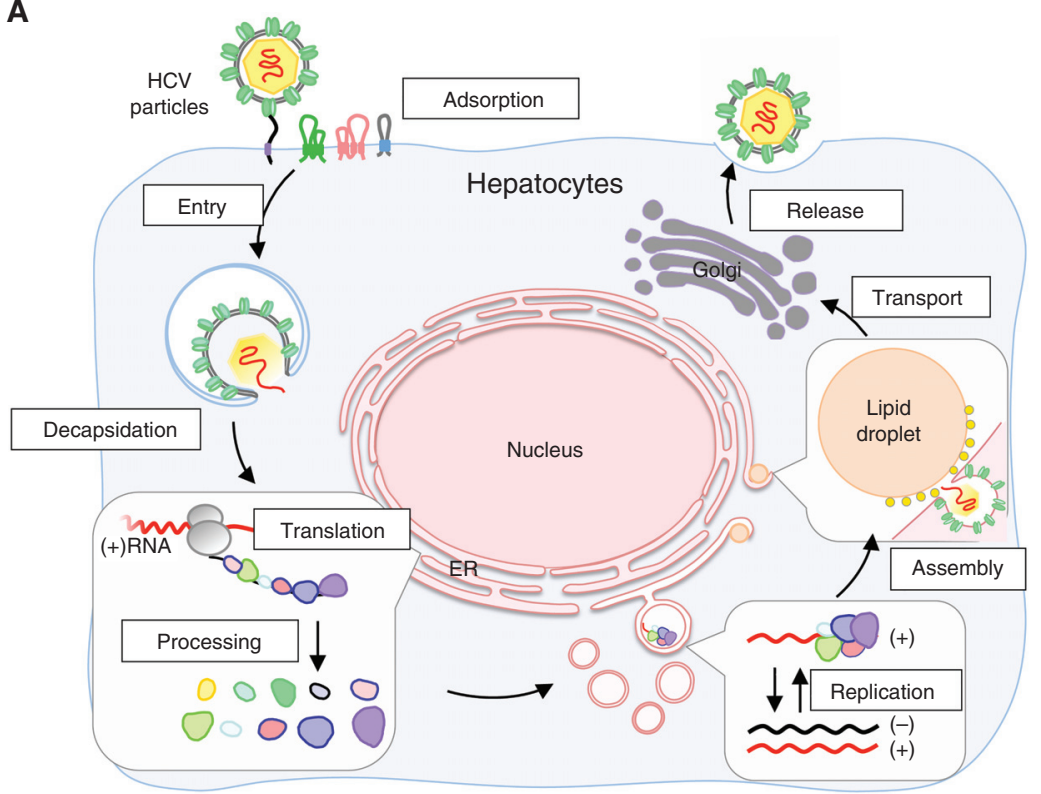

B

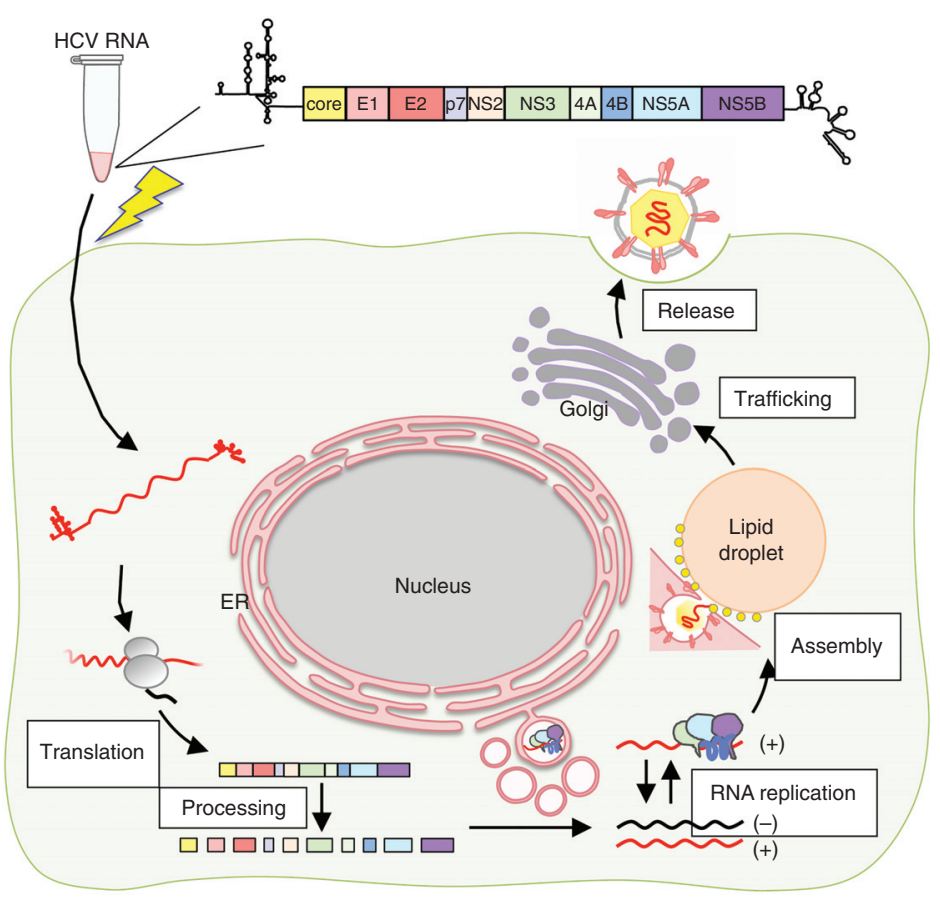

Figure 2. Hepatitis $\mathrm{C}$ virus (HCV) life cycle and HCV cell culture (HCVcc). (A) HCV life cycle. Circulating virus particles bind and then invade the target cells by endocytosis. HCV genomic RNA is released in the cytoplasm and the viral polypeptide is translated. Ten viral proteins are processed from the precursor polypeptide by host and viral proteases. Viral proteins and RNA establish replication complexes that replicate the viral RNA. Replicated viral RNA and structural proteins assemble into viral particles that are secreted into the circulation. (B) In vitrosynthesized HCV RNA from template DNA is transfected (via electroporation; lightning bolt symbol) into cultured cells. Transfected RNA serves as template RNA to drive the HCV life cycle as described in A. After RNA translation and replication starts, $\mathrm{HCV}$ undergoes replication cycles like those seen following virus infection. 
T. Wakita

A

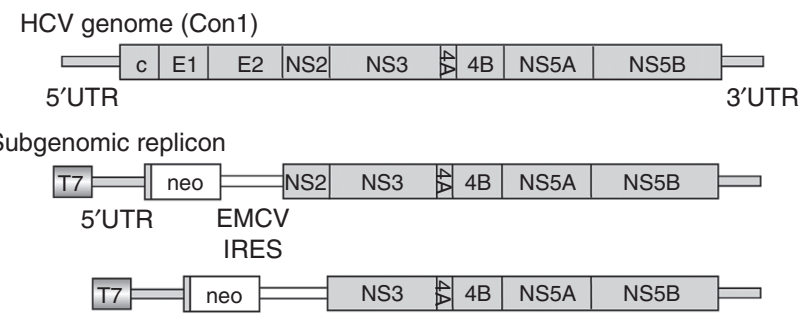

B
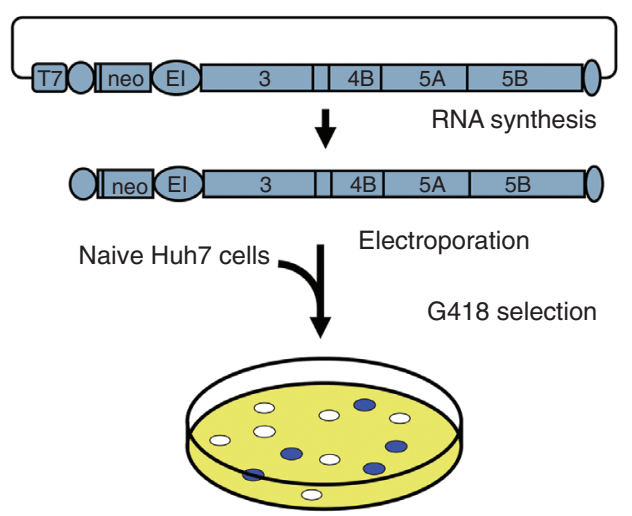

Figure 3. Hepatitis C virus (HCV) replication. (A) First HCV RNA replication in cultured cells. In 1999, Lohmann and colleagues reported the first efficient replication of an HCV subgenomic replicon. These investigators replaced a region encoding several HCV structural proteins (with or without the gene encoding NS2) with a neomycin-resistance-encoding gene (neo). IRES, internal ribosome entry site; EMCV, encephalomyocarditis virus. $(B)$ Subgenomic replicon experiment. Subgenomic replicon RNA was synthesized in vitro and transfected into Huh7 cells by electroporation, followed by G418 selection culture for several weeks, resulting in the establishment of replicons. Robust replication of viral RNA was observed in these cells (Lohmann et al. 1999).

\section{HCV CLONES ISOLATED FROM FULMINANT HEPATITIS PATIENTS AND HCV CULTURE}

The JFH-1 (Jikei or Japanese Fulminant Hepatitis patient) strain was isolated from a 32-yearold male patient (Kato et al. 2001). A distinct strain (JFH-2) was independently isolated from a 62-year-old male treated at a different hospital (Date et al. 2012a). These patients had been admitted with acute liver failure. Stage-II encephalopathy developed in both patients after admission, at which point both were diagnosed with fulminant hepatitis. HCV RNA was detected by RT-PCR using sera obtained during the acute phase. Both patients were seronegative for anti-HCV antibody at admission, but subsequently tested HCV seropositive. All viral markers indicating exposure to other hepatitis viruses were negative. These findings suggested that the fulminant hepatitis in each patient was in fact caused by HCV infection. To analyze the infecting strain of HCV, nested RT-PCR, as well as $5^{\prime}$ rapid amplification of CDNA ends ( $5^{\prime}$-RACE) RT-PCR and 3'-RACE RT-PCR, were performed to cover the entire HCV genome (Wakita 2009). All of the PCR products were cloned and sequenced. Based on sequence analysis, both JFH-1 and JFH-2 belong to genotype $2 \mathrm{a}$, with the nucleotide sequences of the two deviating slightly from other genotype-2a clones isolated from patients with chronic hepatitis (Kato et al. 2001; Date et al. 2012a).

\section{SUBGENOMIC REPLICON USING FULMINANT HCV CLONES}

Subgenomic replicon and full-length constructs were assembled using cloned PCR fragments 
HCV Infection Systems

(Kato et al. 2001; Date et al. 2012a). The colony formation efficiency of the JFH-1 replicon was much greater than that of the JFH-2 replicon. Furthermore, transient transfection of JFH-1 replicon RNA into Huh7 cells resulted in autonomous RNA replication (Kato et al. 2003, 2005). Adaptive mutations were not necessary for efficient JFH-1 replicon replication in Huh7 cells. In contrast, adaptive mutations were detected in the JFH-2 replicon genome, and some of these mutations were shown to enhance replication of the JFH-2 replicon.

\section{FIRST INFECTIOUS HCV CULTURE SYSTEM DEVELOPMENT}

It has been difficult to infect cultured cells with $\mathrm{HCV}$ starting from clinically isolated patient plasma or sera. Although an infectious HCV cDNA was established by in vivo inoculation of synthesized RNA into chimpanzee, attempts to infect cultured cells with those clones were not successful. We found that the JFH-1 strain, despite lacking cell culture-adaptive mutations, showed high levels of subgenomic replicon replication in Huh7 cells (Kato et al. 2003). We therefore attempted direct transfection of Huh7 cells by in vitro-synthesized full-length JFH-1 RNA (Figs. 2B and 4; Wakita et al. 2005; Kato et al. 2006; Wakita 2009). Indeed, transfected full-length JFH-1 RNA replicated efficiently in the cells; secretion of infectious virus particles was confirmed by immunostaining of the infected cells for HCV proteins. In our first experiment, only a small number of the cells were infected. However, this infection was specific, because anti-CD81 or anti-E2 antibodies neutralized infection (Wakita et al. 2005; Zhong et al. 2005). Furthermore, authenticity of the secreted virus particle was proved by the fact the secreted virus particles were infectious in chimpanzee (Wakita et al. 2005; Kato et al 2008). These results strongly suggested that the secreted JFH-1 virus particles were authentic HCV. However, the secretion of infectious viral particles was a characteristic unique to the JFH1 strain; this property has not been reported for the wild-type full-length HCV sequence of any $\mathrm{HCV}$ isolate other than the JFH-1 strain. Furthermore, transfection of either J6/JFH-1 chimeric RNA or JFH-1 RNA into Huh7.5 or Huh7.5.1 cells (both of which are derived from Huh7 cells) yields much higher levels of virus production than that seen with the transfection of Huh7 cells by JFH-1 (Lindenbach et al. 2005; Zhong et al. 2005). Thus, the combination of JFH-1-derived HCV constructs and highly permissive Huh7-derived cells (see below) ensures robust $\mathrm{HCV}$ replication and infection in cell culture.

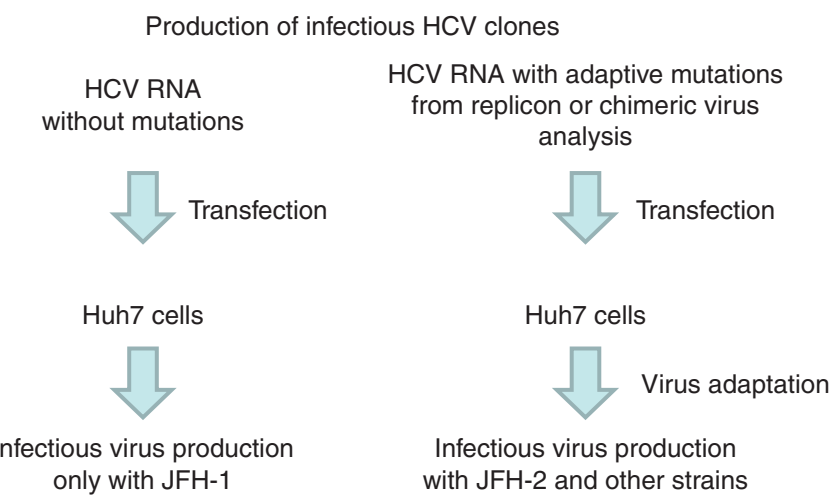

Figure 4. Procedures to establish infectious hepatitis C virus (HCV) clones. Procedures with the JFH-1 clone (left) and other HCV strains (right) are described. JFH-1 required no adaptive mutations to replicate in Huh7 cells; however, efficient replication of other HCV strains required several viral mutations. To identify the key mutations necessary for each HCV strain, replicon analysis or chimeric virus analysis was performed. Furthermore, repeated culturing and passaging of HCV RNA-transfected cells was necessary to establish full cell culture-adapted virus clones. 
T. Wakita

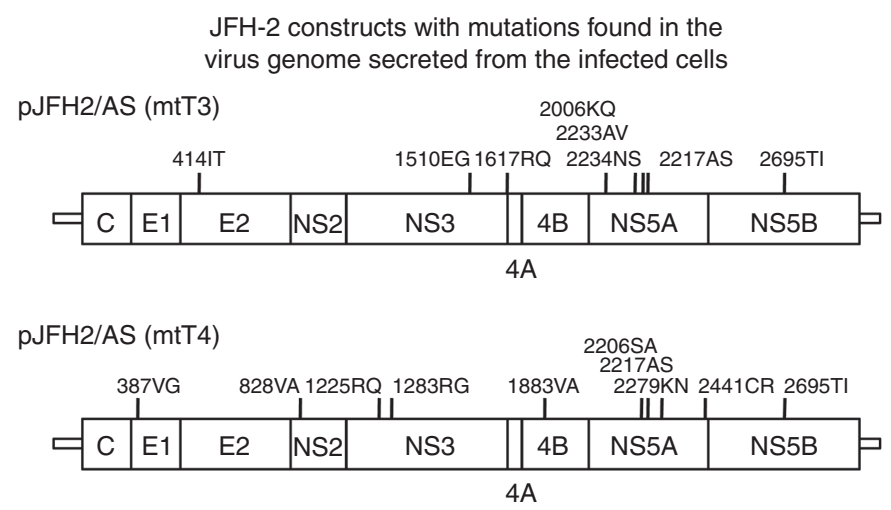

Figure 5. Cell culture-adapted JFH-2 virus genome. Full cell culture-adapted JFH-2 virus genomes were analyzed. Eight and ten adaptive mutations were found in the mtT3 and mtT4 genomes, respectively. These JFH2/AS (mtT3) and JFH2/AS(mtT4) clones were capable of autonomous replication and produced infectious virus in cell culture. Other cell culture-adapted hepatitis $\mathrm{C}$ virus (HCV) strains also possess several adaptive mutations (as summarized in Ramirez and Bukh 2018).

\section{ESTABLISHMENT OF OTHER INFECTIOUS CLONES WITH MUTATIONS OBTAINED FROM THE REPLICON GENOME}

We first hypothesized that JFH-1's capacity for high-level replication in cell culture might reflect the strain's isolation from a patient with fulminant hepatitis. The second fulminant hepatitis isolate, JFH-2, was evaluated in a similar fashion. In contrast to the case with JFH-1, transfection of Huh7 cells with the original (unmutated) full-length JFH-2 RNA did not yield viral replication or virus particle production. We then tested full-length JFH-2 RNA carrying the 2217AS mutation (JFH-2/AS), which was the most efficient adaptive mutation in the replicon experiment. JFH-2/AS virion production was detected for at least $30 \mathrm{~d}$ after transfection. Secreted JFH-2/AS virus particles showed characteristics similar to those of JFH-1 virions, with a notable exception: JFH-2/AS virions lacked in vivo infectivity in human liver-transplanted chimeric mice. In addition, further adaptive mutations were found in the viral genome of the cell culture-adapted JFH-2/AS virus genome (Fig. 5; Date et al. 2012a). Thus, the comparison between the JFH-1 and JFH-2 strains showed that isolation of HCV from fulminant hepatitis cases was not the sole determinant of the culture-competent phenotype. However, based on the JFH-2 strain analysis, we found that some replication-enhancing adaptive mutations permit infectious virus production after transfection of the full-length HCV RNA into Huh7 cells.

In previous reports, adaptive mutations were shown to enhance viral RNA replication at the expense of virus particle formation efficiency (Pietschmann et al. 2002). Specifically, a highly cell culture-adapted Con1 strain replicated in cultured cells but did not produce infectious virus particles. Interestingly, a highly adapted Con1 strain was not infectious for chimpanzee, whereas a moderately adapted Con 1 strain was infectious. However, the virus recovered from the infected animal had the nucleotide sequence of the wild-type Con1 virus (Bukh et al. 2002). This result clearly suggested that HCV strains with lower replication efficiency were favored for in vivo infection. However, we must note that this replication efficiency was determined in cultured cells such as hepatoma cell lines that show gene expression patterns different from those of hepatocytes. In the case of JFH-2, fulllength RNA with the most-adaptive mutation, 2217AS, was able to replicate and produce virus particles. Secreted virus particles were infectious for naive Huh7.5.1 cells, but additional adaptive mutations were found in the viral genomic RNA packaged in these virions. Based on compari- 
sons of cell culture-adapted viruses and their parental virus constructs, adaptive mutations are necessary to increase both viral genome replication and virus particle assembly/secretion efficiency.

Thus, methods for the production of infectious $\mathrm{HCV}$ in cell culture were established (Fig. 4). In the case of JFH-1, these methods are very simple: On transfection of the synthesized full-length HCV RNA into Huh7 cells, virus RNA replication and infectious virus production are observed (Fig. 4, left). However, the replication of other HCV strains requires some modification, notably including the introduction of cell culture-adaptive mutations (Fig. 4, right). The adaptive mutations identified in the subgenomic replicon assay were introduced into the full-length genomes, and the cells transfected with the resulting viral RNA were passaged repeatedly until virus particles were generated. This approach was used successfully to establish a genotype-3a HCV culture system. Genotype 3 is the second most prevalent HCV genotype in Asian countries (after genotype 1b). A high incidence of hepatic steatosis is associated with clinical infection by genotype 3a. Notably, protease inhibitors like telaprevir and boceprevir are less effective against genotype-3a infections (Gottwein et al. 2011). Thus, it is important to study the pathogenesis of genotype- $3 \mathrm{HCV}$ and to assess this genotype's susceptibility to different antiviral inhibitors. We first identified the S310 strain as a full-length genotype-3a HCV cDNA from a 71-year-old female patient suffering from posttransplantation recurrence of $\mathrm{HCV}$ infection. A subgenomic replicon of S310 yielded drug-resistant replicon colonies; sequencing of the resulting replicons revealed the presence of multiple independent adaptive mutations (2186TI, 2188TA, 2198RH, 2496TI, 2895RK, 2895RG, and 2496TI+2895RG) (Saeed et al. 2013). Each of these mutations, as well as the 2210SI mutation (2204SI in genotype 1b), was introduced into the full-length $\mathrm{S} 310 \mathrm{HCV}$ cDNA constructs. When each of the synthesized full-length S310 RNAs with adaptive mutations was transfected into Huh7.5.1 cells, the transfectants produced infectious virus in the cell culture medium (Kim et al. 2014). Virus particles secreted into the culture medium were found to resemble those of other cell culture-adapted viruses. We also compared anti-HCV drug susceptibility between cells infected with S310 and JFH-1. Notably, the protease inhibitor telaprevir appeared to be less effective against S310 infection than against JFH-1 infection, consistent with results previously reported by replicon assay. In contrast, an NS5A inhibitor (PSI-6130), an NS5B inhibitor (BMS-790052), and host-targeting drugs showed similar levels of inhibition of infection by the two viral genotypes. Thus, we successfully established a genotype-3a HCV infectious clone. We also have applied this method to other genotypes, successfully establishing a genotype-4a infectious HCV (Watanabe et al 2018)

A similar approach was taken to establish a genotype-1a infectious HCV clone. A genotype1a H77S strain containing five adaptive mutations was reported to produce infectious virus after transfection of Huh7 cells with a synthesized RNA, albeit with limited efficiency (Yi et al. 2006). H77S.2, H77S.3, and H77-D, subclones that were derived from the adapted H77S clone, showed greater infectivity than the original H77S adapted virus (Shimakami et al. 2011; Yamane et al. 2014). Furthermore, genotype-1a, TNcc, HCV1cc, and H77Ccc infectious clones also were established by the JFH-1 chimeric clone method described below (Li et al. 2015). Although genotype $1 \mathrm{~b}$ is the major subtype in Japan and many other countries, a robust virus culture system is not available for genotype- $1 \mathrm{~b}$ $\mathrm{HCV}$. To address this shortcoming, we isolated an HCV cDNA (designated NC1) from a patient with acute severe hepatitis (Date et al. 2012b). An NC1 subgenomic replicon experiment identified several mutations that enhanced the colony formation efficiency of the NC1 replicon. Interestingly, the full-length $\mathrm{NC1}$ genome with these adaptive mutations replicated in cultured cells and produced infectious virus particles. However, the viral infection efficiency of this clone was not sufficient for autonomous virus propagation in cultured cells. The establishment of a genotype-1b infectious HCV clone remains one of the most important targets of this research field. 
T. Wakita

\section{INFECTIOUS HCV cDNAs DEVELOPED FROM CHIMERIC CONSTRUCTS}

Dr. Jens Bukh's group has established several infectious HCV cDNAs by using chimeric constructs based on the JFH-1 clone (Ramirez and Bukh 2018). Given that HCV strains other than JFH-1 are not infectious and do not replicate in cell culture without adaptive mutations, Bukh's group prepared chimeric constructs using the nonstructural genome of JFH-1. The chimeric synthetic RNAs were transfected into host cells, and the resulting viral genomes were analyzed; the adaptive mutations observed in these isolates were introduced back into the chimeric constructs and into the original HCV strain. Repetition of this cycle resulted in the establishment of infectious HCV clones (Fig. 4, right panel; reviewed in detail in Ramirez and Bukh 2018). Li and colleagues reported that the introduction of three mutations (LSG mutations; located in the NS3-, NS4A-, and NS5B-encoding loci) permitted adaptation and propagation of full-length J6 and J8 (genotype 2b) viruses, yielding clones with infectious titers comparable to those obtained in the JFH-1 system ( $\mathrm{Li}$ et al. 2012a). Introduction of the LSG substitutions also permitted the development of highly infectious versions of TN, HCV-1, and H77C strains (genotype 1a) and of DH8 and DH10 strains (genotype 2b) (Li et al. 2012b, 2015; Ramirez et al. 2014). Isolation of DBN3cc, a genotype-3a infectious HCV clone (Ramirez et al. 2016), and of HK2cc and HK6acc, infectious genotype-6a clones (Pham et al. 2018), were also reported.

Separately, we used a chimeric method to establish an infectious HCV clone. The J6 strain is a prototype genotype-2a HCV strain. J6CF was established as an HCV clone that was infectious in chimpanzee (Yanagi et al. 1997); however, neither full-length nor subgenomic replicons of J6CF are able to replicate in Huh7 cells (Murayama et al. 2007), despite similarities of the J6CF nucleotide sequences to those of JFH-1 across the entire $\mathrm{HCV}$ genome. To address the difference in replicative ability between J6CF and JFH-1, we performed chimeric replicon analysis. The swapping of segments between the two strains revealed that the NS3 helicaseencoding region and the NS5B-encoding region (up to the $3^{\prime} X$ site) were important for efficient replication and virus particle formation by the JFH-1 strain (Murayama et al. 2007).

We subsequently identified the responsible nucleotide or amino acid mutations in the N5BX region of J6CF (Murayama et al. 2010). We assessed the effect of several mutations that introduced JFH-1 $\mathrm{N} 3 \mathrm{H}$ sequences into the J6CF chimeric virus and found that the sequences resulting in the introduction of JFH-1-type amino acids in the NS5B protein (450AS, 517RK, and 561YF) and a nucleotide mutation (9458CG) within the $3^{\prime}$-untranslated region (UTR) were responsible for the replication competency of the chimeric clone. To establish replication-competent J6CF strains with a minimum of changes, we sought to identify the responsible mutations instead of replacing the entire $\mathrm{N} 3 \mathrm{H}$ region. We attempted to find the responsible residues acids in the $\mathrm{N} 3 \mathrm{H}$ region by replacing a partial region of $\mathrm{N} 3 \mathrm{H}$ but could not identify the responsible region or specific amino acids. Therefore, we exploited the strategy of long-term culture of chimeric constructs with low replication efficiencies (Murayama et al. 2017). For this purpose, we used the J6CF chimeric virus J6/5BSLX-JFH1, which carries JFH-1 predicted stem-loop structures from the NS5B (5BSL)-to- $3^{\prime} X$ region. This J6/ 5BSLX-JFH1 chimeric virus replicated efficiently in cell culture when combined with a $\mathrm{N} 3 \mathrm{H}$ replacement but showed very low replication efficiency without the $\mathrm{N} 3 \mathrm{H}$ replacement. Using this strategy, we were able to identify the essential amino acid substitutions that conferred replication competency to J6CF in combination with the previously identified three amino acid substitutions and single nucleotide mutation. Surprisingly, the identified mutation was not in NS3 but in NS4A. Thus, we identified effective cell culture-adaptive mutations and established a replication-competent J6CF strain with minimum modifications in cultured cells (Murayama et al. 2017). However, the robust replication efficiency of the JFH-1 strain may be the result of the enzymatic activities of NS3 and NS5B. In fact, in vitro polymerase 
activity of JFH-1 NS5B was higher than that of J6 NS5B (Schmitt et al. 2011). Interestingly, structural analysis of JFH-1 NS5B revealed that a critical amino acid, 405I, enhances de novo initiation. Recently, the NS3 protein from the JFH-1 strain has been found to show robust helicase activity compared with the NS3 protein from genotype 1b (Zhou et al. 2017). Helicase activity, which is important in virus RNA replication, should be compared between JFH-1 and J6 strains.

\section{PERMISSIVE CELL LINES}

Although the infection efficiency of JFH-1 virus in the original Huh7 cells maintained in our laboratory was quite low, efficient viral infection was achieved using "cured" cells, such as Huh7.5, Huh7.5.1, and Lunet/CD81 cells (Blight et al. 2002; Zhong et al. 2005; Koutsoudakis et al. 2007). These cell lines were "cured" by interferon treatment of cells carrying subgenomic replicons. Huh7.5 cells harbor a point mutation in the gene encoding RIG-I, resulting in defective intracellular interferon signaling in response to HCV RNA replication (Sumpter et al. 2005). Transfection of the JFH-1 genome or inoculation of infectious JFH-1 virus into Huh7.5 and Huh7.5.1 cells thus results in robust replication and HCV virus infection (Lindenbach et al. 2005; Zhong et al. 2005). Koutsoudakis and colleagues have reported that the level of CD81 cell surface expression is a key determinant of $\mathrm{HCV}$ infection (Koutsoudakis et al. 2007). Huh7 cells were first isolated more than 20 years ago (Nakabayashi et al. 1982) and have been subsequently distributed worldwide. The phenotype of Huh7 cells, including permissiveness for JFH-1 virus infectivity, likely varies among the subclones maintained in different laboratories. Recently, Saeed et al. (2015) reported that SEC14L2 is an important host factor that enables $\mathrm{HCV}$ clones with wild-type sequences to replicate in cultured cells. SEC14L2 is expressed in human liver but not in Huh7 cells. Therefore, Huh7 cells that stably express SEC14L2 were prepared and used to establish HCV replicon cell with wild-type HCV clones. SEC14L2-expressing
Huh7 cells that express one or more additional host factor(s) may provide an excellent opportunity to develop clinical HCV infection systems in the near future.

\section{CONCLUSIONS}

A robust $\mathrm{HCV}$ infection system was first reported in 2005; the resulting isolate, the JFH-1 strain, remains the only HCV strain that can be propagated in Huh7 cells in the absence of adaptive mutations. This system has been useful for multiple purposes in the HCV research field, including the development of antivirals and vaccines, as well as improved characterization of the $\mathrm{HCV}$ viral life cycle. Infection systems for $\mathrm{HCV}$ of other genotypes also have been developed in recent years. Future studies should be directed to establish robust infectivity, especially for genotype- $1 \mathrm{~b}$, and clinical HCV isolates.

\section{ACKNOWLEDGMENTS}

The author thanks Dr. Senko Tsukuda for her help with the illustrations. This study was supported by the Japan Society for the Promotion of Science KAKENHI (Grant No. JP17K09447) and by the Japan Agency for Medical Research and Development, AMED (Grant Nos. JP18fk 0210009j0003 and JP18fk0210002j10031).

\section{REFERENCES}

Bartenschlager R, Lohmann V. 2001. Novel cell culture systems for the hepatitis $C$ virus. Antiviral Res 52: 1-17. doi:10.1016/S0166-3542(01)00164-4

Blight KJ, Kolykhalov AA, Rice CM. 2000. Efficient initiation of HCV RNA replication in cell culture. Science 290: 1972-1974. doi:10.1126/science.290.5498.1972

Blight KJ, McKeating JA, Rice CM. 2002. Highly permissive cell lines for subgenomic and genomic hepatitis $\mathrm{C}$ virus RNA replication. J Virol 76: 13001-13014. doi:10.1128/ JVI.76.24.13001-13014.2002

Blight KJ, McKeating JA, Marcotrigiano J, Rice CM. 2003. Efficient replication of hepatitis $\mathrm{C}$ virus genotype 1a RNAs in cell culture. J Virol 77: 3181-3190. doi:10 .1128/JVI.77.5.3181-3190.2003

Bukh J, Pietschmann T, Lohmann V, Krieger N, Faulk K, Engle RE, Govindarajan S, Shapiro M, St. Claire M, Bartenschlager R. 2002. Mutations that permit efficient replication of hepatitis $\mathrm{C}$ virus RNA in Huh-7 cells prevent productive replication in chimpanzees. Proc Natl Acad Sci 99: 14416-14421. doi:10.1073/pnas.212532699 
T. Wakita

Choo QL, Kuo G, Weiner AJ, Overby LR, Bradley DW, Houghton M. 1989. Isolation of a cDNA clone derived from a blood-borne non-A, non-B viral hepatitis genome. Science 244: 359-362. doi:10.1126/science.2523562

Date T, Kato T, Kato J, Takahashi H, Morikawa K, Akazawa D, Murayama A, Tanaka-Kaneko K, Sata T, Tanaka Y, et al. 2012a. Novel cell culture-adapted genotype 2a hepatitis C virus infectious clone. J Virol 86: 10805-10820. doi:10.1128/JVI.07235-11

Date T, Morikawa K, Tanaka Y, Tanaka-Kaneko K, Sata T, Mizokami M, Wakita T. 2012b. Replication and infectivity of a novel genotype $1 \mathrm{~b}$ hepatitis $\mathrm{C}$ virus clone. Microbiol Immunol 56: 308-317. doi:10.1111/j.1348-0421.2012 $.00437 . \mathrm{x}$

Gottwein JM, Scheel TK, Jensen TB, Ghanem L, Bukh J. 2011. Differential efficacy of protease inhibitors against HCV genotypes $2 \mathrm{a}, 3 \mathrm{a}, 5 \mathrm{a}$, and $6 \mathrm{a}$ NS3/4A protease recombinant viruses. Gastroenterology 141: 1067-1079. doi:10.1053/j.gastro.2011.06.004

Hartlage AS, Cullen JM, Kapoor A. 2016. The strange, expanding world of animal hepaciviruses. Annu Rev Virol 3: 53-75. doi:10.1146/annurev-virology-100114-055104

Ikeda M, Yi M, Li K, Lemon SM. 2002. Selectable subgenomic and genome-length dicistronic RNAs derived from an infectious molecular clone of the HCV-N strain of hepatitis C virus replicate efficiently in cultured Huh7 cells. J Virol 76: 2997-3006. doi:10.1128/JVI.76.6.29973006.2002

Kato T, Furusaka A, Miyamoto M, Date T, Yasui K, Hiramoto J, Nagayama K, Tanaka T, Wakita T. 2001. Sequence analysis of hepatitis $\mathrm{C}$ virus isolated from a fulminant hepatitis patient. J Med Virol 64: 334-339. doi:10.1002/ jmv.1055

Kato T, Date T, Miyamoto M, Furusaka A, Tokushige K, Mizokami M, Wakita T. 2003. Efficient replication of the genotype 2a hepatitis $\mathrm{C}$ virus subgenomic replicon. Gastroenterology 125: 1808-1817. doi:10.1053/j.gastro 2003.09.023

Kato T, Date T, Miyamoto M, Sugiyama M, Tanaka Y, Orito E, Ohno T, Sugihara K, Hasegawa I, Fujiwara K, et al. 2005. Detection of anti-hepatitis $C$ virus effects of interferon and ribavirin by a sensitive replicon system. J Clin Microbiol 43: 5679-5684. doi:10.1128/JCM.43.11.56795684.2005

Kato T, Date T, Murayama A, Morikawa K, Akazawa D, Wakita T. 2006. Cell culture and infection system for hepatitis C virus. Nat Protoc 1: 2334-2339. doi:10.1038/ nprot.2006.395

Kato T, Choi Y, Elmowalid G, Sapp RK, Barth H, Furusaka A, Mishiro S, Wakita T, Krawczynski K, Liang TJ. 2008. Hepatitis $\mathrm{C}$ virus JFH-1 strain infection in chimpanzees is associated with low pathogenicity and emergence of an adaptive mutation. Hepatology 48: 732-740. doi:10.1002/ hep. 22422

Kim S, Date T, Yokokawa H, Kono T, Aizaki H, Maurel P, Gondeau C, Wakita T. 2014. Development of hepatitis C virus genotype 3a cell culture system. Hepatology 60: 1838-1850. doi:10.1002/hep.27197

Koutsoudakis G, Herrmann E, Kallis S, Bartenschlager R, Pietschmann T. 2007. The level of CD81 cell surface expression is a key determinant for productive entry of hep- atitis C virus into host cells. J Virol 81: 588-598. doi:10 .1128/JVI.01534-06

Krieger N, Lohmann V, Bartenschlager R. 2001. Enhancement of hepatitis $C$ virus RNA replication by cell cultureadaptive mutations. J Virol 75: 4614-4624. doi:10.1128/ JVI.75.10.4614-4624.2001

Kuo G, Choo QL, Alter HJ, Gitnick GL, Redeker AG, Purcell RH, Miyamura T, Dienstag JL, Alter MJ, Stevens CE, et al. 1989. An assay for circulating antibodies to a major etiologic virus of human non-A, non-B hepatitis. Science 244: 362-364. doi:10.1126/science. 2496467

Lanford RE, Lee H, Chavez D, Guerra B, Brasky KM. 2001. Infectious cDNA clone of the hepatitis $C$ virus genotype 1 prototype sequence. J Gen Virol 82: 1291-1297. doi:10 1099/0022-1317-82-6-1291

Li YP, Ramirez S, Gottwein JM, Scheel TK, Mikkelsen L, Purcell RH, Bukh J. 2012a. Robust full-length hepatitis $\mathrm{C}$ virus genotype $2 \mathrm{a}$ and $2 \mathrm{~b}$ infectious cultures using mutations identified by a systematic approach applicable to patient strains. Proc Natl Acad Sci 109: E1101-E1110. doi:10.1073/pnas.1203829109

Li YP, Ramirez S, Jensen SB, Purcell RH, Gottwein JM, Bukh J. 2012b. Highly efficient full-length hepatitis $C$ virus genotype 1 (strain TN) infectious culture system. Proc Natl Acad Sci 109: 19757-19762. doi:10.1073/pnas .1218260109

Li YP, Ramirez S, Mikkelsen L, Bukh J. 2015. Efficient infectious cell culture systems of the hepatitis $\mathrm{C}$ virus (HCV) prototype strains HCV-1 and H77. J Virol 89: 811-823. doi:10.1128/JVI.02877-14

Lindenbach BD, Evans MJ, Syder AJ, Wolk B, Tellinghuisen TL, Liu CC, Maruyama T, Hynes RO, Burton DR, McKeating JA, et al. 2005. Complete replication of hepatitis C virus in cell culture. Science 309: 623-626. doi:10 $.1126 /$ science. 1114016

Lohmann V, Körner F, Koch J, Herian U, Theilmann L, Bartenschlager R. 1999. Replication of subgenomic hepatitis C virus RNAs in a hepatoma cell line. Science $\mathbf{2 8 5}$ 110-113. doi:10.1126/science.285.5424.110

Lohmann V, Korner F, Dobierzewska A, Bartenschlager R. 2001. Mutations in hepatitis $C$ virus RNAs conferring cell culture adaptation. J Virol 75: 1437-1449. doi:10.1128/ JVI.75.3.1437-1449.2001

Murayama A, Date T, Morikawa K, Akazawa D, Miyamoto M, Kaga M, Ishii K, Suzuki T, Kato T, Mizokami M, et al 2007. The NS3 helicase and NS5B-to- $3^{\prime} \mathrm{X}$ regions are important for efficient hepatitis $\mathrm{C}$ virus strain JFH-1 replication in Huh7 cells. J Virol 81: 8030-8040. doi:10.1128/ JVI.02088-06

Murayama A, Weng L, Date T, Akazawa D, Gao Y, Chen W, Suzuki T, Kato T, Mizokami M, Wakita T, et al. 2010 Specific RNA structures and mutations implicated for HCV RNA replication and virus particle formation in cultured cells. PLoS Pathog 6: e1000885.

Murayama A, Sugiyama N, Suzuki R, Moriyama M, Nakamura N, Mochizuki H, Wakita T, Kato T. 2017. Amino acid mutations in the NS4A region of hepatitis $\mathrm{C}$ virus contribute to viral replication and infectious virus production. J Virol 91: e02124-16. doi:10.1128/JVI.02124-16

Nakabayashi H, Taketa K, Miyano K, Yamane T, Sato J. 1982. Growth of human hepatoma cells lines with differentiated 
HCV Infection Systems

functions in chemically defined medium. Cancer Res 42: 3858-3863.

Pham LV, Ramirez S, Gottwein JM, Fahnøe U, Li YP, Pedersen J, Bukh J. 2018. HCV genotype 6a escape from and resistance to velpatasvir, pibrentasvir, and sofosbuvir in robust infectious cell culture models. Gastroenterology 154: 2194-2208.e12. doi:10.1053/j.gastro.2018.02.017

Pietschmann T, Lohmann V, Kaul A, Krieger N, Rinck G, Rutter G, Strand D, Bartenschlager R. 2002. Persistent and transient replication of full-length hepatitis $C$ virus genomes in cell culture. J Virol 76: 4008-4021. doi:10 $.1128 / J V I .76 .8 .4008-4021.2002$

Ramirez S, Bukh J. 2018. Current status and future development of infectious cell-culture models for the major genotypes of hepatitis $\mathrm{C}$ virus: Essential tools in testing of antivirals and emerging vaccine strategies. Antiviral Res 158: 264-287. doi:10.1016/j.antiviral.2018.07.014

Ramirez S, Li YP, Jensen SB, Pedersen J, Gottwein JM, Bukh J. 2014. Highly efficient infectious cell culture of three hepatitis $\mathrm{C}$ virus genotype $2 \mathrm{~b}$ strains and sensitivity to lead protease, nonstructural protein $5 \mathrm{~A}$, and polymerase inhibitors. Hepatology 59: 395-407. doi:10.1002/hep .26660

Ramirez S, Mikkelsen LS, Gottwein JM, Bukh J. 2016. Robust HCV genotype 3a infectious cell culture system permits identification of escape variants with resistance to sofosbuvir. Gastroenterology 151: 973-985.e2. doi:10.1053/j .gastro.2016.07.013

Saeed M, Gondeau C, Hmwe S, Yokokawa H, Date T, Suzuki T, Kato T, Maurel P, Wakita T. 2013. Replication of hepatitis $\mathrm{C}$ virus genotype $3 \mathrm{a}$ in cultured cells. Gastroenterology 144: 56-58.e7. doi:10.1053/j.gastro.2012.09.017

Saeed M, Andreo U, Chung HY, Espiritu C, Branch AD, Silva JM, Rice CM. 2015. SEC14L2 enables pan-genotype HCV replication in cell culture. Nature 524: 471-475. doi:10.1038/nature14899

Schmitt M, Scrima N, Radujkovic D, Caillet-Saguy C, Simister PC, Friebe P, Wicht O, Klein R, Bartenschlager R, Lohmann V, et al. 2011. A comprehensive structure-function comparison of hepatitis C virus strain JFH1 and J6 polymerases reveals a key residue stimulating replication in cell culture across genotypes. J Virol 85: 2565-2581. doi:10.1128/JVI.02177-10

Shimakami T, Welsch C, Yamane D, McGivern DR, Yi M, Zeuzem S, Lemon SM. 2011. Protease inhibitor-resistant hepatitis $\mathrm{C}$ virus mutants with reduced fitness from impaired production of infectious virus. Gastroenterology 140: 667-675. doi:10.1053/j.gastro.2010.10.056
Sumpter R Jr, Loo YM, Foy E, Li K, Yoneyama M, Fujita T, Lemon SM, Gale M Jr. 2005. Regulating intracellular antiviral defense and permissiveness to hepatitis $\mathrm{C}$ virus RNA replication through a cellular RNA helicase, RIGI. J Virol 79: 2689-2699.

Wakita T. 2009. Isolation of JFH-1 strain and development of an HCV infection system. Methods Mol Biol 510: 305327. doi:10.1007/978-1-59745-394-3_23

Wakita T, Pietschmann T, Kato T, Date T, Miyamoto M, Zhao Z, Murthy K, Habermann A, Krausslich HG, Mizokami M, et al. 2005. Production of infectious hepatitis $\mathrm{C}$ virus in tissue culture from a cloned viral genome. Nat Med 11: 791-796. doi:10.1038/nm1268

Watanabe N, Suzuki T, Date T, Hmwe SS, Aly HH, Aizaki H, Sugiyama M, Mizokami M, Kassas ME, Tabl A, et al. 2018. Establishment of infectious genotype 4a HCVcc. Presented at 25th International Symposium on Hepatitis C Virus and Related Viruses. Dublin, Ireland, October 8 11, 2018.

Yamane D, McGivern DR, Wauthier E, Yi M, Madden VJ, Welsch C, Antes I, Wen Y, Chugh PE, McGee CE, et al. 2014. Regulation of the hepatitis $C$ virus RNA replicase by endogenous lipid peroxidation. Nat Med 20: 927-935. doi:10.1038/nm.3610

Yanagi M, Purcell RH, Emerson SU, Bukh J. 1997. Transcripts from a single full-length cDNA clone of hepatitis $\mathrm{C}$ virus are infectious when directly transfected into the liver of a chimpanzee. Proc Natl Acad Sci 94: 8738-8743. doi:10.1073/pnas.94.16.8738

Yanagi M, Purcell RH, Emerson SU, Bukh J. 1999. Hepatitis C virus: An infectious molecular clone of a second major genotype (2a) and lack of viability of intertypic 1a and 2a chimeras. Virology 262: 250-263. doi:10.1006/viro.1999.9889

Yi M, Villanueva RA, Thomas DL, Wakita T, Lemon SM. 2006. Production of infectious genotype 1a hepatitis C virus (Hutchinson strain) in cultured human hepatoma cells. Proc Natl Acad Sci 103: 2310-2315. doi:10.1073/ pnas.0510727103

Zhong J, Gastaminza P, Cheng G, Kapadia S, Kato T, Burton DR, Wieland SF, Uprichard SL, Wakita T, Chisari FV. 2005. Robust hepatitis C virus infection in vitro. Proc Natl Acad Sci 102: 9294-9299. doi:10.1073/pnas .0503596102

Zhou T, Ren X, Adams RL, Pyle AM. 2017. NS3 from hepatitis $\mathrm{C}$ virus strain JFH-1 is an unusually robust helicase that is primed to bind and unwind viral RNA. J Virol 92: e01253-17. 


\section{$\&_{\mathrm{CSH}}^{\infty} \&$ Cold Spring Harbor

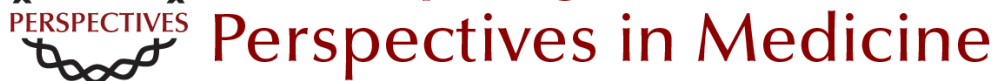

\section{Cell Culture Systems of HCV Using JFH-1 and Other Strains}

Takaji Wakita

Cold Spring Harb Perspect Med 2019; doi: 10.1101/cshperspect.a036806 originally published online September 9, 2019

\section{Subject Collection Hepatitis C Virus: The Story of a Scientific and Therapeutic Revolution}

Antibody Responses in Hepatitis C Infection Mansun Law

T-Cell Immunity against the Hepatitis C Virus: A Persistent Research Priority in an Era of Highly Effective Therapy

Stephanie Smith, Jonathan R. Honegger and Christopher Walker

Interferon-Free Hepatitis C Virus Therapy Jean-Michel Pawlotsky

Animal Models of Hepatitis C Virus Infection Alexander Ploss and Amit Kapoor

Natural History of Hepatic and Extrahepatic Hepatitis C Virus Diseases and Impact of Interferon-Free HCV Therapy

Francesco Negro

Hepatitis C Virus Epidemiology and the Impact of Interferon-Free Hepatitis C Virus Therapy Jeffrey V. Lazarus, Elena Roel and Ahmed M. Elsharkawy

Hepatitis C Virus Entry: Protein Interactions and Fusion Determinants Governing Productive Hepatocyte Invasion

Gisa Gerold, Rebecca Moeller and Thomas Pietschmann

Hepatitis C Virus Structure: Defined by What It Is Not

Altaira D. Dearborn and Joseph Marcotrigiano
Innate Immunity in Hepatitis C Virus Infection Johannes Schwerk, Amina Negash, Ram Savan, et al.

HCV Assembly and Egress via Modifications in Host Lipid Metabolic Systems Kunitada Shimotohno

Control of HCV Infection by Natural Killer Cells and Macrophages

Hugo R. Rosen and Lucy Golden-Mason

The Elimination of Hepatitis $\mathbf{C}$ as a Public Health

Threat Margaret Hellard, Sophia E. Schroeder, Alisa Pedrana, et al.

Hepatitis C Virus Replication Keisuke Tabata, Christopher J. Neufeldt and Ralf Bartenschlager

Challenges and Promise of a Hepatitis C Virus Vaccine Andrea L. Cox

Rewiring Host Signaling: Hepatitis C Virus in Liver Pathogenesis

Alessia Virzì, Armando Andres Roca Suarez, Thomas F. Baumert, et al.

Hepatitis C Virus: $\mathbf{3 0}$ Years after Its Discovery Michael Houghton

For additional articles in this collection, see http://perspectivesinmedicine.cshlp.org/cgi/collection/ 